\title{
EDITORIAL
}

\section{Formación de profesionales de enfermería bajo el contexto de los modelos teóricos}

i partimos de la premisa de que un modelo no se ajusta a la realidad, entonces no se puede asegurar que una teoría
de enfermería representa en realidad la práctica del cuidado. Este razonamiento no tiene una conclusión definida, sin
embargo, nos invita a reflexionar sobre el uso de modelos y teorías en enfermería. El propósito de este manuscrito es
realizar una breve reflexión sobre la formación de profesionales de enfermería, el uso y aplicación de modelos y teorías
en la práctica e investigación.

\section{¿Qué es un modelo de enfermería?}

Existen varias definiciones, pero si consideramos a Jacqueline Fawcett, un modelo teórico es "un conjunto de conceptos abstractos y generales y las proposiciones que expresan algo acerca de los conceptos; dada la naturaleza abstracta y general de los conceptos, las ideas, que describen o vinculan los conceptos, también son abstractas y generales" (1).

Luego entonces, si un modelo es la representación conceptual de lo que se construye alrededor del fenómeno del cuidado de enfermería, su nivel es muy abstracto. Es decir, el grado de utilidad de los conceptos de cada teoría puede funcionar o no para cada situación en particular, dado que la forma en que se puede medir dicho concepto estriba entre la abstracción a un nivel filosófico a un indicador empírico que demuestre que el concepto es medible.

Así que, tenemos una disyuntiva, por un lado, existen varios fenómenos del cuidado que son sumamente subjetivos que no tienen forma de cuantificarse y carecen de instrumentos de medición, es aquí donde están los modelos que algunos profesionales los consideran aún filosofías del cuidado, por ejemplo, teóricas como Henderson, Watson, Abdellah, King, entre otras. Sin embargo, a pesar de su poca utilidad en ciertos campos reales de la práctica del cuidado, estos modelos parecen ideales para iniciar la formación de los profesionales de enfermería, porque dentro de sus enunciados teóricos prevalece el sentido original del cuidado, que es el respeto por la vida del ser humano, por su salud y enfermedad, por el ambiente y la comunidad.

Por otro lado, hay modelos con ideales deterministas, que a pesar de tener muy bien definidos sus constructos, sus postulados y axiomas, no se ajustan a la realidad. Estos modelos son los más utilizados en la investigación, pues tienen instrumentos que pueden probar sus postulados teóricos, indican causalidad y variaciones, también se pueden someter a modelos matemáticos, pero en muchos casos (la mayoría) se tienen que complementar con modelos teóricos de otras disciplinas para poder explicar el fenómeno de estudio, situación conocida como teorías prestadas, que es el caso de la mayoría de las teorías de enfermería (no hay una originalidad completa).

A pesar de esto, la utilización de los modelos teóricos en enfermería prevalece, ya que según Jacqueline Fawcett existe una relación (teórica) íntima y bidireccional entre estos y la práctica enfermera (clínica en la mayoría de los casos), en donde ambos se influyen, se transforman y evolucionan para mejorar los estándares de cuidado para cada fenómeno en la realidad (1).

\section{Diferentes ámbitos en la utilización de teorías de enfermería}

Al parecer, el uso de las teorías de enfermería se encuentra en tres niveles, en lo formativo, en la práctica e investigación.

Nivel formativo. En los Estados Unidos de Norte América, los modelos solo son utilizados en este nivel, en las universidades, pero no se utilizan en la práctica clínica profesional, solo en algunos casos para investigación. En América Latina, la situación no es diferente, por ejemplo, en el caso de Colombia y México, diversas Instituciones de Educación Superior (IES), muestran en sus mallas curriculares la utilización de modelos y teorías de enfermería, ya sea para un semestre en particular o para guiar las valoraciones durante todas las unidades de aprendizaje. Entre los más utilizados en Colombia está el Modelo de Watson y Roy, en México se encuentran a Henderson y Orem; sin embargo, no utilizan todo el Modelo, porque son muy amplios y generales, son más utilizados para guiar la valoración de enfermería. Por ejemplo, en México se utilizan las 14 necesidades o los Requisitos Universales y en algunas IES también se utilizan los patrones funcionales de Marjory Gordon y así, explicar y guiar la primera fase del Proceso de Atención de Enfermería (PAE).

Nivel práctico. Existen pocos casos documentados donde las instituciones de salud (fuera de un ámbito universitario) han implementado la utilización de los modelos teóricos en las notas de enfermería y en los planes de cuidado. En la Secretaría de Salud, los más conocidos son la utilización de la teoría de Virginia Henderson en el Instituto Nacional de Cardiología lgnacio Chávez y la utilización del Modelo del Déficit de Autocuidado de Orem en el Hospital General de México Dr. Eduardo Liceaga; incluso en instituciones particulares como el Hospital Ángeles, se publicó en la revista Desarrollo Científico de Enfermería un 
modelo ecléctico de estas dos teóricas. Ninguno de los tres casos o ninguno otro caso documentado, ha logrado establecer un modelo teórico como guía en la atención de los usuarios en instituciones de salud de manera oficial, solo se pueden leer propuestas y estudios pilotos en servicios de salud específicos.

En México, la tendencia actual es la propuesta de un modelo ecléctico construido por algunas nomenclaturas americanas (NANDA-NIC-NOC), se incluyen las bases del paradigma de enfermería descrito por Chin y Kramer(2), el PAE con cinco etapas y la utilización de Guías de Práctica Clínica; pero, sin la utilización de un modelo teórico de enfermería en particular.

Nivel de investigación. En general es amplio el uso de los modelos teóricos de enfermería en el ámbito de la investigación, se utilizan en los niveles de posgrado en enfermería de todos los programas en México. En la mayoría de los casos para probar relaciones teóricas entre variables o conceptos, también se utilizan como guía para la construcción de estudios de caso; sin embargo, aunque su uso académico prevalece, ha disminuido su utilización en los últimos años, de acuerdo con García y cols. ${ }^{(3)}$, del año 2005 al 2010, se publicaron 358 artículos de investigación de enfermería en revistas mexicanas, identificando que el 57.8\% de los artículos no utilizaron teoría de enfermería, es decir, 207 artículos. Si comparamos con un segundo corte publicado en el año 2018, donde se identificaron 244 artículos de investigación publicados en las mismas revistas mexicanas del año 2010 al 2015, el 84\% de los artículos no utilizaron teoría de enfermería; en otras palabras, "solo 40 trabajos de investigación utilizaron teoría en un periodo de 5 años". Ante la evidencia del análisis de estas revisiones se puede deducir que en un periodo de 10 años el uso de modelos teóricos en México disminuyó 26.2\%, por lo tanto, en los siguientes 20 años es posible que disminuya $52.4 \%{ }^{(4)}$.

\section{Comentarios finales}

No se puede asegurar que un modelo se ajusta perfectamente a la realidad, los modelos de enfermería pueden ser útiles, pero no representan la realidad de la practica de enfermería, por lo tanto, la mayoría de ellos tienen que ser complementados con otros conceptos teóricos de diferentes disciplinas.

Retomar teorías que no tienen elementos prestados de otras disciplinas como los Modelos del Déficit de Autocuidado de Orem y la Teoría de las Transiciones de Afaf Meleis, fortalecería la formación de profesionales de enfermería, para el ámbito clínico o comunitario y para la investigación, sin embargo, no abarcan situaciones específicas.

Para lo anterior, una alternativa es fomentar el uso de las teorías de situaciones especificas (las mal llamadas microteorías), las cuales son teorías de menor abstracción claramente diseñadas para situaciones especificas, por lo que se hace menos complejo llevarlas a la practica real de enfermería; tal es el caso de la Teoría de Síntomas Desagradables de Lenz, la Teoría de la Incertidumbre de Mishel, Teoría del autocuidado en la falla cardíaca de Riegel, entre otras.

Los modelos teóricos de enfermería en México tuvieron gran importancia en la década de los años noventa y al principio de este siglo. La enseñanza académica de la enfermería en la práctica e investigación han tenido un respaldo con la visión de las teorías, sin embargo, persiste la brecha entre la teoría y la práctica, así el sistema de salud mexicano ha apostado por un modelo más práctico, menos teórico y basado en evidencia científica publicada, no en postulados que están dispuestos a probarse, sólo el tiempo dirá que fue lo mejor.

\section{Erick Landeros-Olvera'; Natalia Ramírez-Girón ${ }^{2}$}

${ }^{1}$ Doctor en Ciencias de Enfermería. Miembro del Sistema Nacional de Investigadores del CONACyT. Facultad de Enfermería, Benemérita Universidad Autónoma de Puebla.E-mail: erick_landeross@hotmail.com

2Maestra en Enfermería. Estudiante de Doctorado en Ciencias de Enfermería. Facultad de Enfermería, Universidad Autónoma de Nuevo León.

\section{REFERENCIAS BIBLIOGRÁFICAS}

1.Fawcett J. Analysis and evaluation of conceptual models Philadelphia: F.A. Davis Company; 1995. 576 p.

2.Chinn P, Kramer M. Theory and nursing. 5th ed. St. Louis: Mosby; 1999.

3.García-Rodríguez M, Gómez-Ávila M, Aguilar-Pérez I, Pérez-Martínez G, Velázquez-Díaz L, Soriano-Sotomayor M, et al. Tendencias y características de la investigación en enfermería publicada en México. Enfermería Universitaria. 2011; 8(1): 7-16.

4.Landeros-Olvera E, Ramírez-Girón N, Yáñez-Lozano Á, Guzmán-Ramírez G, Galicia-Aguilar R. Temáticas y características metodológicas de la investigación de enfermería publicada en revistas mexicanas: 2010-2015. Enfermería Universitaria. 2018; 15(3). 